\title{
APORTACIONES DE LA ARQUEOLOGÍA SUBACUÁTICA AL PATRIMONIO ORGANOLÓGICO
}

\section{CONTRIBUTIONS OF THE UNDERWATER ARCHEOLOGY TO THE ORGANOLOGICAL HERITAGE}

Jesús Alonso Yllana

Escuela Municipal de Música y Danza de Pozuelo de Alarcón (Madrid)

\section{Resumen}

El presente artículo se centra en el estudio de los restos de instrumentos musicales hallados en excavaciones arqueológicas subacuáticas en diferentes partes del mundo. Esos restos no han sido objeto de estudio hasta la fecha, exceptuando alguna investigación parcial.

En primer lugar se catalogan, posibilitando así que el musicólogo tenga acceso a estos documentos que tienen la ventaja de no haber sufrido modificaciones de épocas posteriores como sucede en muchos casos en los instrumentos conservados en tierra. Se establece así una vía de comunicación efectiva entre el trabajo de los arqueólogos subacuáticos y los musicólogos.

En segundo lugar, se expone el estudio de uno de los instrumentos catalogados que por su importancia histórica es digno de destacar entre los hallados. Dicho estudio consiste en un análisis de los restos y un estudio musicológico sobre el instrumento.

\section{Palabras clave}

Musicología, arqueología subacuática, organología, instrumentos musicales, guitarra de cuatro órdenes.

Los trabajos históricos -en musicología- se basan en el estudio de la documentación escrita, de los instrumentos musicales y de los documentos sonoros. Hoy en día la disponibilidad de nuevos medios tecnológicos facilita el desarrollo de disciplinas que aportan información a la que anteriormente no se tenía acceso. Esta situación obliga a una gran labor de coordinación entre diferentes áreas de trabajo.

Cuando el arqueólogo subacuático George Bass desarrolla sus primeros trabajos en Turquía en la década de los 60 y comienza a utilizar técnicas de trabajo análogas a las

\begin{abstract}
The present article focuses on the study of musical instruments remains found in underwater archaeological excavations in different parts of the world which so far were in a state of almost total lack of study.

Firstly, they are cataloged, providing musicologists access to these documents. These instruments have the advantage of not having undergone any changes over time, which is not the case with the instruments preserved ashore, as many of them suffered subsequent changes. This line of work establishes a channel of effective communication between the work of underwater archaeologists and musicologists.

Secondly, one of the instruments listed, a remarkable one due to its historical significance, has been thoroughly studied. This study is an analysis of the remains and musicological study of the instrument.
\end{abstract}

Key words

Musicology, underwater archaeology, organology, musical instruments, four-course guitar.

empleadas en tierra, la arqueología subacuática comienza a tener un desarrollo creciente. A partir de entonces es el arqueólogo el supervisor y director de los trabajos que comprenden aspectos interdisciplinarios de la investigación: buzos profesionales, manejo de equipos sofisticados o personal cualificado en diversas disciplinas.

Las excavaciones arqueológicas de yacimientos subacuáticos nos ofrecen informaciones clave sobre centros de tráfico y rutas comerciales, intercambio de saberes e interrelación artística y cultural en diferentes épocas. 
Los excelentes trabajos realizados desde hace varias décadas en arqueología subacuática, han dado como fruto la aparición de patrimonio histórico que estaba guardado sin haber sido modificado, tanto por el paso de los años como por la acción de los hombres de generaciones posteriores.

El altísimo valor en todo orden de aspectos y la singular belleza de los objetos traídos a la luz del día en numerosas excavaciones hace que estemos obligados a valorar el esfuerzo económico y humano que se deriva de estos trabajos. Debemos ordenar dentro de cada área de conocimiento los documentos que la arqueología subacuática nos ofrece y aprovechar la información que estos documentos nos apor$\tan$.

Si bien ya han sido realizados interesantes estudios en otras materias relacionadas con la arqueología subacuática - por ejemplo la evolución de la construcción naval-, son todavía inexistentes los trabajos sistemáticos sobre instrumentos musicales encontrados en excavaciones, los cuales no han sido estudiados ni catalogados.

La arqueología y la organología deben conseguir que la interpretación de los datos se produzca de manera natural en este camino conjunto que se abre entre los arqueólogos y los musicólogos.

Uno de los objetivos que nos planteamos conseguir con este trabajo es agilizar para los arqueólogos el acceso a la musicología. Los organólogos podrán así realizar análisis de piezas que no han tenido un estudio adecuado hasta este momento.

Los instrumentos musicales que han estado a disposición de los estudiosos, han llegado hasta nuestra época de forma casual. En muchos casos la dificultad de conservación de los materiales orgánicos ha contribuido a la desaparición de gran parte de ellos. Esto induce a pensar que en un ambiente acuático y especialmente en uno marino, estas condiciones ambientales eliminan la posibilidad de obtener documentos en este ámbito. Además, el desconocimiento de la importante función social que la música desarrolló en épocas anteriores en los barcos ha propiciado un total desinterés por el acercamiento de los musicólogos a los resultados de los trabajos de arqueología subacuática.

En este momento, es posible el estudio de interesantes documentos que nos hablan de la relación entre la música y la mar. Ambas han sido compañeras inseparables desde tiempos inmemoriales en la actividad marinera y los naufragios nos han legado en ocasiones restos de instrumentos musicales. ${ }^{1}$

1 Sobre este asunto disponemos de un artículo de gran interés escrito por REY (2004): 95-140.
Por todo esto, tras varios meses de establecer contactos con personas, grupos de estudio y organismos que tienen relación con el mundo de la arqueología subacuática vimos que no eran una casualidad las primeras referencias que se habían encontrado. Se podía intentar seguir el rastro de más hallazgos que después de estar ocultos en las aguas seguían ocultos en polvorientas estanterías de centros de estudio arqueológico o que se podían observar en vitrinas de museos sirviendo únicamente como curiosidades para ocasionales visitantes pero sin haber tenido ningún tipo de estudio.

Por esto, se ha hecho una búsqueda sistemática contactando con museos, laboratorios y centros en todo el mundo que tienen relación con la arqueología subacuática. La pretensión de tal búsqueda ha sido encontrar el mayor número de restos de instrumentos musicales y objetos sonoros que, provenientes de excavaciones en medio subacuático, pudieran formar un corpus digno de clasificar.

Con esta perspectiva, la primera decisión fue hacer una catalogación lo más amplia posible de hallazgos — tanto instrumentos musicales como objetos sonoros-, tuvieran o no algún estudio hecho que los acompañara tras su descubrimiento, su extracción de las aguas y su tratamiento en el laboratorio.

Como resultado se han catalogado restos de instrumentos musicales provenientes de excavaciones realizadas en diferentes partes del mundo desde el segundo tercio del s. $x x^{2}$ Esta catalogación pretende también la inclusión de toda la información procedente de las excavaciones arqueológicas subacuáticas que en el futuro se vayan produciendo y cuyo destino lógico sea el de los estudios organológicos adecuados.

Podemos así, estudiar piezas procedentes de excavaciones que llevan a reconocer y a posibilitar el estudio de instrumentos de música antiguos, sus formas, características o los materiales utilizados en su construcción. Cada descubrimiento de un instrumento musical o de fragmentos aporta un cierto número de informaciones (dadas por las trazas de uso o de fabricación, la estratigrafía, el contexto en el que se ha hallado, etc.) y al mismo tiempo suscita una investigación documental complementaria basada en la iconografía, los textos y los instrumentos contemporáneos.

Los restos encontrados abren un camino que puede aportar con el paso del tiempo importantes datos que vengan a ampliar el conocimiento de la evolución de algunos instrumentos musicales.

2 La catalogación, así como los diversos aspectos de la investigación expuestos en este artículo, se pueden consultar en ALONSO YLLANA (2013). 
El proceso que sería ideal cumplir comienza en la información que el metódico trabajo de los arqueólogos subacuáticos nos proporciona. El organólogo se debe encargar del estudio y análisis de los restos. Con los resultados, el constructor de instrumentos puede hacer una reproducción en la que poner a prueba, por un lado, los datos que el musicólogo extrae de los documentos escritos y, por otro, el desarrollo de la actividad interpretativa llevada a cabo por el intérprete. ${ }^{3}$

Podemos así, evitar el fracaso que supone el no estudiar en profundidad restos que ha costado -a las administraciones y a los arqueólogos- gran cantidad de dinero y esfuerzo recuperar.

En los archivos se nos plantea también un largo e interesante trabajo. Se podría mejorar el conocimiento de las vías de expansión musical, mediante el análisis y la catalogación sistemática de los viajes y sus motivaciones para embarcar instrumentos a bordo. Esto también ampliaría el punto de vista sobre las posibilidades de presencia de instrumentos de música en los barcos. Se precisa también pues, un trabajo profundo y sistemático en los archivos para ampliar las fronteras de la investigación organológica.

Como sabemos, en muchos de los instrumentos que tenemos actualmente en colecciones o museos, se han introducido modificaciones estructurales y no se han conservado algunos elementos. Curiosamente los restos de algunos instrumentos provenientes de excavaciones subacuáticas desvelan algunos de estos aspectos.

Se recogen en este artículo las piezas de 24 excavaciones diferentes con 120 registros catalográficos en total. Se han realizado consultas a personalidades, diferentes especialistas, responsables de excavaciones, museos navales o marítimos, departamentos de universidades, comités, ministerios de cultura, centros e institutos de arqueología marítima, centros de conservación de objetos históricos, instituciones, responsables de colecciones, grupos de investigación, asociaciones y, en general, a todos los entes a los que se ha tenido acceso y que pudieran tener alguna relación posible con los restos arqueológicos que se han extraído de excavaciones arqueológicas subacuáticas. El abanico ha sido muy amplio, pero estamos seguros de que queda mucho trabajo por hacer. Incluso contactando con un centro donde pueda haber restos de interés, no siempre se habla con la persona adecuada. Esto supone que no son conocedores o no recuerdan en detalle los miles de restos que custodian y que además en muchos casos no están convenientemente catalogados. Por otro lado, se han establecido contactos con más de una veintena de países:
Alemania, Australia, Austria, Bélgica, Canadá, Bulgaria, Dinamarca, Estados Unidos, Irlanda, República Dominicana, Rusia, España, Finlandia, Francia, Grecia, India, Italia, Estonia, Ecuador, Holanda, Noruega, Nueva Zelanda, Reino Unido, Suecia, Portugal, Malasia, Turquía y Sudáfrica.

Se reúnen pues, piezas de épocas muy alejadas en el tiempo, en el espacio y de ámbitos culturales muy diferentes. Esto da una idea de la gran variedad de tipologías y procedencias de las piezas estudiadas. Pretende por eso esta catalogación convertirse en una herramienta de trabajo para investigadores de muy diferentes campos dentro de la musicología y una base de trabajo para el estudio profundo y centrado en familias instrumentales, épocas diferentes o la ampliación de conocimientos de algún instrumento concreto en el que se tenga especial interés y dispongamos de algún ejemplar.

Así, sería ideal que cada una de las piezas que aquí encontramos llegase a ser estudiada y valorada por especialistas que sepan incluirlos adecuadamente en la función musical que desempeñaban en su entorno cultural y social, estudiando sus particularidades históricas, formales, funcionales, evolutivas y acústicas. Podremos recuperarlos como documentos históricos que puedan servir incluso para ser reconstruidos y ayuden a estudiar nuevos aspectos sobre la práctica musical de su época. Aunque en la mayoría de los casos será imposible la total recuperación de la información sobre el ejemplar en concreto, cualquier información parcial, por mínima que sea, deberá ser recogida sistemáticamente y podrá tener un gran valor histórico.

El trabajo realizado presenta los restos de instrumentos en una relación ordenada desde el punto de vista organológico, en fichas individualizadas para ofrecer una información básica que permita abordar nuevas investigaciones. Esta relación está vertebrada según las cuatro grandes clases instrumentales establecidas por Sach y Hornbostel y de acuerdo a los distintos modos de producción del sonido: aerófonos, cordófonos, idiófonos y membranófonos. ${ }^{4}$

Se ha añadido una miscelánea donde se recogen tanto elementos sueltos que participan en la producción del sonido (baquetas, etc), como elementos sin función acústica (clavijas, boquillas, etc) o aquellos en los que no ha sido posible la identificación de su nombre genérico. También hallaremos una sección de iconografía encontrada con elementos musicales (tallas en madera, piezas de cerámica, etc).

Entre paréntesis se especifica el barco en el que viajaba cada instrumento y la fecha en la que se hundió.

4 SACHS - HORNBOSTEL (1914). 


\section{RELACIÓN DE INSTRUMENTOS MUSICALES Y OBJETOS SONOROS}

Aerófonos

Bisel, sin canal: Flautas traveseras

1. Flauta travesera (St. George, 1811)

2. Pífano (Maple Leaf, 1864)

3. Flauta travesera (Maple Leaf, 1864)

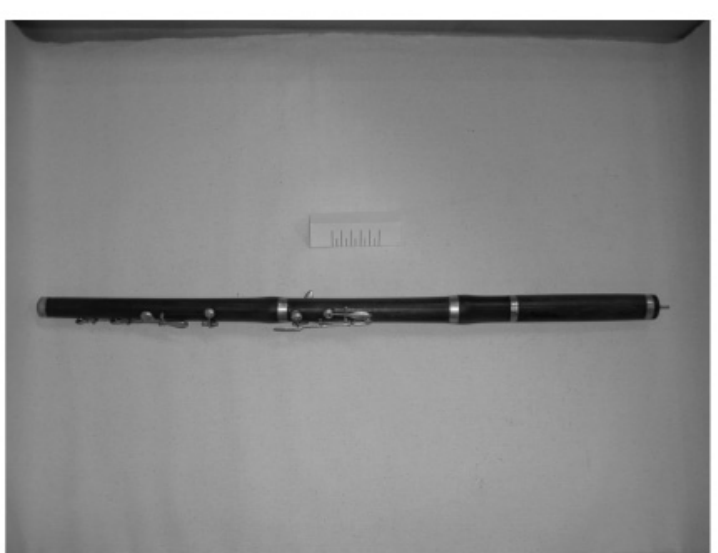

Figura 1. Flauta travesera (Maple Leaf, 1864).
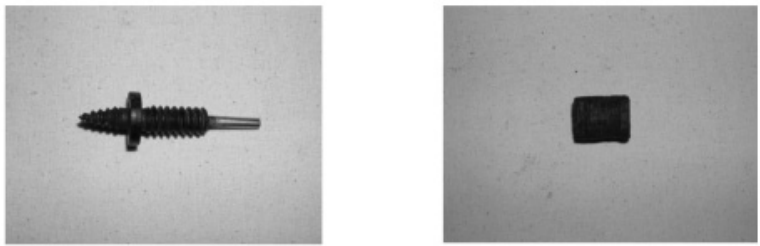

Figura 2. Detalles de piezas de la flauta de la figura 1.

Bisel, con canal: Flautas rectas

4. Flauta de tres agujeros (Mary Rose, 1545)

5. Flauta de tres agujeros (Mary Rose, 1545)

6. Flauta de tres agujeros (y estuche) (Mary Rose, 1545)

7. Flauta (The Queen of Sweden, 1745)

8. Flauta de pico (General Carleton of Whitby, 1785)

9. Flauta de pico (General Carleton of Whitby, 1785)

10. Flageolet (St. George, 1811)

11. Flageolet (St. George, 1811)

Bisel, con canal: Silbatos

12. Silbato de contramaestre (Mary Rose, 1545)

13. Silbato de contramaestre (Mary Rose, 1545)

14. Silbato (Mary Rose, 1545)

15. Silbato (Mary Rose, 1545)

16. Silbato (Mary Rose, 1545)

17. Silbato (Mary Rose, 1545)
18. Silbato (Mary Rose, 1545)

19. Silbato de señales (Conde de Tolosa, 1724)

20. Silbato con cuerpo globular de cerámica (Tek Sing, 1822)

Lengüeta doble

21. Chirimía (Charavines, s. XI)

22. Chirimía (Mary Rose, 1545)

23. Oboe (Nuestra Señora de Guadalupe, 1724)

Lengüeta simple

24. Chalumeau doble (Charavines, s. XI)

25. Flageolet (Charavines, s. XI)

26. Chalumeau (Charavines, s. XI)

27. Clarinete (Maple Leaf, 1864)

28. Clarinete (Maple Leaf, 1864)

29. Clarinete (Maple Leaf, 1864)

Trompetas naturales

30. Caracola de señales (Culip Iv, 69-79d.C.)

31. Caracola de señales (Ha'aheo o Hawaii, 1824)

Boquilla. Sin válvulas

32. Trompeta (Kronan, 1676) 
33. Trompeta (Kronan, 1676)

Cordófonos

Cuerda pulsada

34. Cistro (Trinidad Valencera, 1588)

35. Cistro (Beurtschip "B71", c. 1625)

36. Cistro (Beurtschip "B71", c. 1625)

37. Cistro (Beurtschip "B71", c. 1625)

38. Cistro (Beurtschip "B71", c. 1625)

39. Cistro (Beurtschip "B71", c. 1625)

40. Cistro (Beurtschip "B71", c. 1625)

41. Guitarra (Flota de Nueva España, 1733)

42. Guitarra (Flota de Nueva España, 1733)

43. Guitarra (Flota de Nueva España, 1733)

44. Guitarra (Flota de Nueva España, 1733)

Frotados con arco

45. Fídula (Mary Rose, 1545)

46. Fídula (Mary Rose, 1545)

47. Fídula (Mary Rose, 1545)

48. Vihuela de arco (Kronan, 1676)

49. Vihuela de arco (Kronan, 1676)

50. Vihuela de arco (Kronan, 1676)

51. Vihuela de arco (Kronan, 1676)

52. Vihuela de arco (Kronan, 1676)

53. Vihuela de arco (Kronan, 1676)

54. Violín (Kronan, 1676)

55. Violín (Kronan, 1676)

56. Violín (Kronan, 1676)

57. Violín (Kronan, 1676)

58. Violín (Maple Leaf, 1864)

59. Violín (Maple Leaf, 1864)

60. Violín (Maple Leaf, 1864)

Idiófonos

Golpe directo: Campana

61. Campana (Ciudad de Heraclion, ss. VI-II a.C.)

62. Campana (Ciudad de Heraclion, ss. VI-II a.C.)

63. Campana (Mary Rose, 1545)

64. Campana (Mary Rose, 1545)

65. Campana (San Salvador de Buarcos, 1555)

66. Campana (Kronan, 1676)

67. Campana (Queen Anne's Revenge, 1718)

68. Campana (Queen Anne's Revenge, 1718)

69. Campana (Conde de Tolosa, 1724)

70. Campana (General Carleton of Whitby, 1785)

71. Campana (St. George, 1811)

Golpe indirecto: Sistro

72. Sistro (Ciudad de Heraclion, ss. VI-II a.C.)

73. Sistro (Ciudad de Heraclion, ss. VI-II a.C.)

74. Sistro (Ciudad de Heraclion, ss. VI-II a.C.)

75. Cascabeles (Nuestra Señora de Guadalupe,
Pulsación: Guimbarda

76. Guimbarda o arpa de boca (Queen Anne's Revenge, 1718)

77. Guimbarda o arpa de boca (PS Clonmel, 1841)

Membranófonos

Golpeado. Bimembranófono: Tambor

78. Tamboril (Mary Rose, 1545)

79. Tamboril (Mary Rose, 1545)

80. Caja (Kronan, 1676)

81. Caja (Kronan, 1676)

82. Caja y par de baquetas (St. George, 1811)

Golpeado. Unimembranófono: Tambor

83. Tambor (Charavines, $\mathrm{s}$. XI)

84. Pandereta (Trinidad Valencera, 1588)

Miscelánea

85. Puente de cordófono (Charavines, s. XI)

86. Puente de cordófono (Charavines, s. XI)

87. Clavija de cordófono (Charavines, s. XI)

88. Fondo de cordófono (Charavines, s. XI)

89. Baqueta de tamboril (Mary Rose, 1545)

90. Clavija (Emanuel Point Ship, 1559)

91. Arco de vihuela de arco (Kronan, 1676)

92. ¿Arco? (Kronan, 1676)

93. Arco de violín (Kronan, 1676)

94. Arco de violín (Kronan, 1676)

95. Dos clavijas de cordófono (Kronan, 1676)

96. ¿Arco? (Kronan, 1676)

97. Clavija de cordófono (Kronan, 1676)

98. Dos baquetas (Kronan, 1676)

99. Boquilla de aerófono (Flota de 1715, 1715)

100. Clavija de cordófono (Flota de 1715, 1715)

101. Dos clavijas de cordófono (Flota de Nueva España, 1733)

102. Clavija de cordófono (Flota de Nueva España, 1733) 1811)

103. Clavija y fragmento no determinado (St. George,

104. Resina (Maple Leaf, 1864)

105. Arco de violín (Maple Leaf, 1864)

Iconografía

106. Talla en madera de un arpa (Duart Point, 1653)

107. Guitarra. Miniaturas de cerámica (El Nuevo Constante, 1766)

108. Guitarra. Miniaturas de cerámica (El Nuevo Constante, 1766)

109. Violín. Miniatura de cerámica (El Nuevo Constante, 1766)

110. Talla en madera de Rey David con arpa (Vasa, 1628) 1724) 
111. Talla en madera de ángel con violochelo (Vasa, 1628)

112. Talla en madera de ángel con flauta (Vasa, 1628)

113. Talla en madera de ángel con gaita (Vasa, 1628)

114. Talla en madera de ángel con laúd (Vasa, 1628)

115. Talla en madera de ángel con violín (Vasa, 1628)

116. Talla en madera de ángel con arpa (Vasa, 1628)

117. Talla en madera de ángel con laúd (Vasa, 1628)

118. Talla en madera con trompeta (Vasa, 1628)

119. Talla en madera con serpentón (Vasa, 1628)

120. Talla en madera con serpentón (Vasa, 1628)

El conjunto de todas estas piezas - restos de instrumentos musicales, objetos sonoros y piezas iconográficas - , se ha ordenado siguiendo básicamente los criterios comúnmente utilizados en la catalogación instrumental con algunas variantes para facilitar la búsqueda del material y adaptarlo a las peculiaridades de esta catalogación.

Esta relación, que incluye las denominaciones recibidas como punto de partida, nos sirve para describir los primeros pasos de la metodología desarrollada y que nos llevará desde el hallazgo, la denominación y las impresiones iniciales hasta la definición y las conclusiones que tras el estudio riguroso de una pieza se pueden obtener.
Presentamos el modelo seguido en cada una de las fichas individualizadas de catalogación mediante un ejemplo concreto: los restos del instrumento número 58.

\section{NÚMERO DE REGISTRO CATALOGRÁFICO: 58}

Clase instrumental: Cordófono

Subdivisión: Frotados con arco

Nombre genérico: Violín

Estado de conservación: Incompleto.

Descripción: Fragmentos de un violín. Se conservan restos de cuerda en el clavijero. La barra armónica en la tapa parece estar tallada en la misma tapa. Los aros van insertados en el taco.

Procedencia (yacimiento): Maple Leaf

Fecha del hundimiento: 1864

Lugar de conservación, museo o colección: Florida Bureau of Archaeological Research (B.A.R.), Tallahassee, Florida, EEUU

Número de inventario: $8 \mathrm{MO}-137$, Contract S-7, 95.47.1536

Observaciones: St. John's Archaeological Expeditions, Inc. (SJAEI)
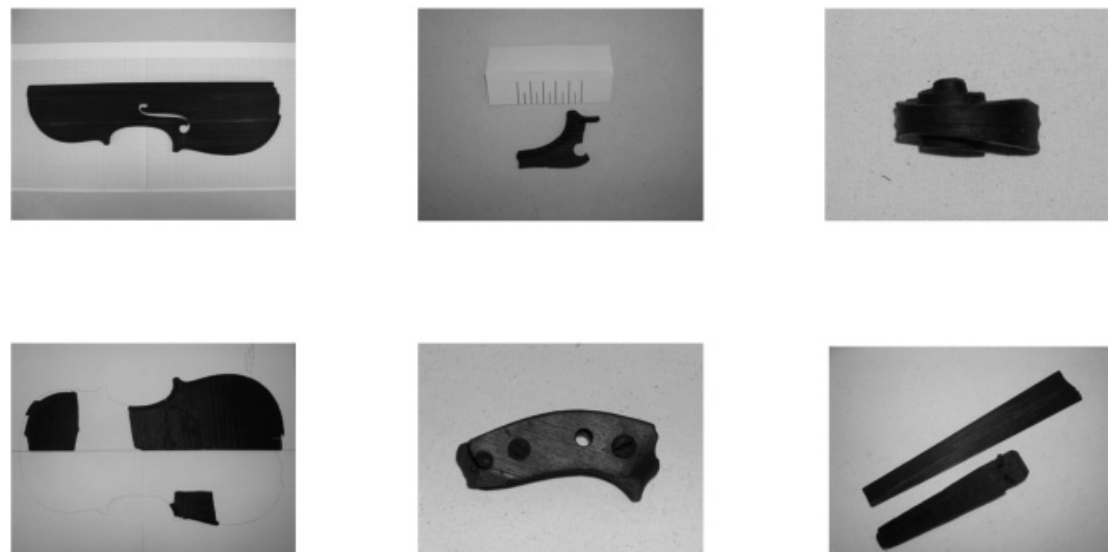

Figura 3. Documentación fotográfica del registro catalográfico ${ }^{\circ} 58$

Partiendo de los restos de al menos tres guitarras de cuatro órdenes incluidos en la catalogación efectuada, hemos desarrollado un modelo de estudio de restos arqueológicos de instrumentos musicales. ${ }^{5}$

5 Registros catalográficos números 41, 42, 43 y 44.
Tras las primeras sospechas de estar tratando con un modelo de guitarra de cuatro órdenes, se ha estudiado en profundidad toda la información que conservamos en la actualidad sobre este instrumento. La evolución de los instrumentos depende siempre de la búsqueda a nivel estético llevada a cabo por el músico y de las mejoras técnicas desarrolladas por el constructor. Por esto, debemos contemplar 
cada ejemplar como un documento absolutamente ligado a su uso. No es completo el estudio sin relacionarlo con su música y su entorno musical.

La historia documentalmente conocida hasta el momento de la guitarra de cuatro órdenes comienza en el siglo XVI, concretamente en 1546 en Sevilla. ${ }^{6}$ De dicho siglo conservamos música escrita en tablatura, descripciones formales, de la afinación, y una cantidad de datos que permiten intuir bastantes aspectos de su descripción. Desgraciadamente no conservamos ejemplares originales de ese período que puedan confirmar o desmentir lo expresado en los documentos de los que disponemos.

Se examinan las guitarras de cuatro órdenes que han llegado hasta nuestros días para establecer una comparativa y una valoración del ejemplar que motiva este estudio. La investigación interdisciplinaria debe también contar obligatoriamente con algunas disciplinas como la iconografía. Extraemos en cada caso los elementos organológicos de interés. El caso que nos ocupa es muy interesante pues los modelos originales no existían por el momento y disponemos ahora de estos restos que aportan nueva información.

Para poder seguir adelante con la interpretación de los restos en estudio, hemos recurrido a la reconstrucción de un instrumento del cual no disponemos de originales. Esto supone abrir la posibilidad de encajar muchos datos de los que se disponía pero que por sí solos no tenían la capacidad de darnos una visión muy certera de algunos detalles. Para ello, se ha procedido a hacer un estudio de cada una de las piezas por separado, realizando dibujos para recomponer las formas de estos restos. (Figuras. 4-8)

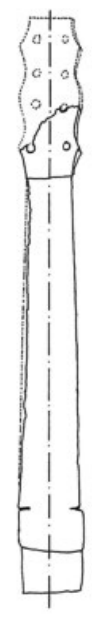

Figura 6. Pieza ${ }^{\circ} 42$

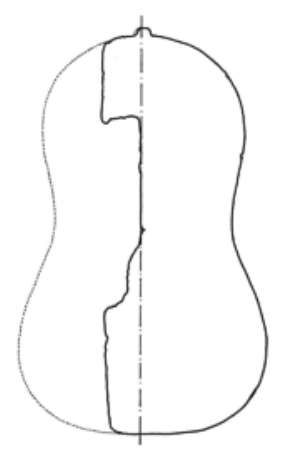

Figura 7. Pieza $\mathrm{n}^{\circ} 44$

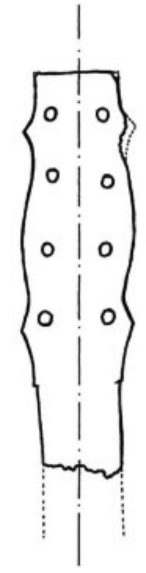

Figura 4. Pieza ${ }^{\circ} 43$

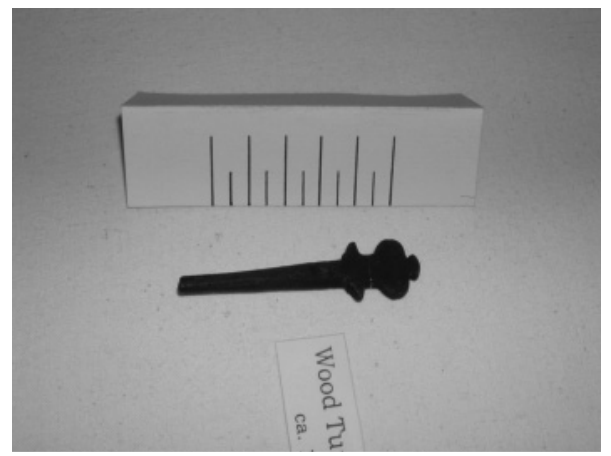

Figura 5. Pieza $\mathrm{n}^{\circ} 102$

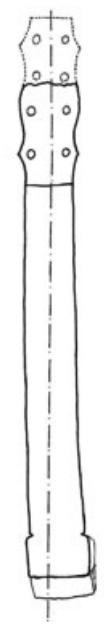

Figura 8. Pieza $\mathrm{n}^{\circ} 41$

6 ARRIAGA, 1/2 (Madrid, 1988): 369-406. 
Tras terminar los planos necesarios, se inicia el proceso de reconstrucción de los restos arqueológicos cuyo modelo resultante podemos observar en la siguiente ilustración.

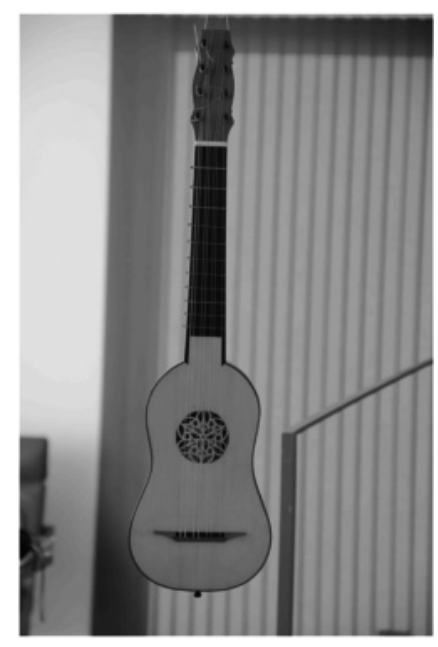

Figura 9. Resultado de la reconstrucción

La reconstrucción del instrumento (Figura 9) al que pertenecen los restos arqueológicos ha hecho posible el estudio de la música que fue escrita para la guitarra de cuatro órdenes y que ha llegado hasta nuestros días. Así, se ha podido determinar de manera práctica el grado de adaptación del instrumento reconstruido al repertorio citado.

Las coincidencias de los datos extraídos de toda la documentación examinada dibujan un perfil del instrumento - en aspectos como el ámbito, el tamaño, la proporción, las características formales o la afinación - que se ajusta plenamente con el resultante del estudio de las piezas originales y la referida reconstrucción.

Tres aspectos fundamentales en su función musical -la interválica entre los órdenes, la altura de afinación, y los intervalos entre las cuerdas de cada orden- se cumplen incluso analizando los diferentes ámbitos conocidos (se puede ajustar a los diapasones utilizados en la música Europea en diferentes períodos, países y contextos musicales). El tiro concuerda con las descripciones y permite el uso musical requerido por las fuentes musicales del siglo xvi. Así, tras la construcción de un prototipo que cumple con las características estudiadas en los restos arqueológicos se ha podido observar que la música conservada para la guitarra de cuatro órdenes es perfectamente practicable en el modelo llevado a cabo.

Desgraciadamente no ha sido posible su datación dendrocronológica, que nos daría por lo menos una fecha antes de la cual no pudieron haberse construido los instrumentos estudiados. Convendría señalar que mientras no se realice esa prueba $-\mathrm{y}$ todo indica que no se podrá realizar-, el tamaño y la factura de las guitarras concuerdan con lo que sabemos de ellas en el siglo XVI por otras fuentes. Los restos estudiados aportan nueva información o la confirmación de datos que no conocíamos en profundidad en el conocimiento de la evolución de la guitarra en general.

Ante todos estos resultados, se puede concluir que nos encontramos ante los fragmentos de al menos tres ejemplares de un modelo español de guitarra de cuatro órdenes. Por lo tanto, aunque no sepamos su fecha de construcción, sí podemos decir con certeza que son los únicos restos conocidos de una guitarra de cuatro órdenes que concuerdan con todos los datos que nos ofrece la documentación del siglo xvi, por lo que no sería extraño que pertenecieran a un instrumento de esa época.

Las opciones tomadas como punto de partida no han de ser necesariamente aceptadas totalmente. Éstas generarán variedad de opiniones que deben contribuir positivamente al deseo de avanzar. Dicha diversidad puede contribuir a cambiar el método de trabajo cuando sea necesario y a rebatirlo mediante la muestra de resultados concretos. La metodología que se ha desarrollado se podría aplicar a cualquiera de los restos incluidos en la catalogación, adaptándolo lógicamente a las características singulares de cada caso.

\section{BIBLIOGRAFÍA}

Alonso Yllana, Jesús: "Fragmentos de guitarras de cuatro órdenes procedentes de un naufragio", Roseta, $\mathrm{n}^{\circ} 5$, (2010), pp. 24-33.

Alonso Yllana, Jesús: “Contribución de la arqueología subacuática al conocimiento de la evolución de la guitarra", tesis doctoral, Universidad Complutense Madrid, 2013.

Arriaga, Gerardo: "La guitarra de cuatro órdenes en el siglo XVI", Música y Educación, 1/2, (1988), pp. 369-406.

Rey, Pepe: "Apuntes sobre música naval y náutica", Javier Suárez Pajares, John Griffiths (eds.), Políticas y prácticas musicales en el mundo de Felipe II: estudios sobre la música en España, sus instituciones y sus territorios en la segunda mitad del siglo XVI, Madrid: Universidad Complutense, Instituto Complutense de Ciencias Musicales, 2004, pp. 95-140.

Sachs, Curt; y Hornbostel, Erich M. von: "Systematik der Musikinstrumente. Ein Versuch", Zeitschrift für Ethnologie, $\mathrm{n}^{\mathrm{o}} 46,(1914)$, pp. 553-590.

Recibido: 14.05 .2015 Aceptado: 03.05.2016 